\title{
New feasible iterative algorithms and strong convergence theorems for bilevel split equilibrium problems
}

\section{Zhenhua $\mathrm{He}^{1}$ and Wei-Shih Du${ }^{2 *}$}

\section{"Correspondence:}

wsdu@nknucc.nknu.edu.tw

${ }^{2}$ Department of Mathematics,

National Kaohsiung Normal

University, Kaohsiung, 824, Taiwan

Full list of author information is

available at the end of the article

\begin{abstract}
In this paper, we first introduce and investigate a bilevel split equilibrium problem (BSEP) which can be regarded as a new development in the field of equilibrium problems. We provide some new feasible iterative algorithms for BSEP and establish strong convergence theorems for these iterative algorithms in different spaces. MSC: 47J25; 47H09;65K10

Keywords: metric projection; adjoint operator; equilibrium problem (EP); split equilibrium problem (SEP); bilevel split equilibrium problem (BSEP); bilevel convex optimization problem (BCOP); the common solution of equilibrium problems (CEP); feasible iterative algorithm; strong convergence theorem
\end{abstract}

\section{Introduction and preliminaries}

Let $K$ be a closed convex subset of a real Hilbert space $H$. Let $\langle\cdot, \cdot\rangle$ and $\|\cdot\|$ denote the inner product of $H$ and the norm of $H$, respectively. For each point $x \in H$, there exists a unique nearest point in $K$, denoted by $P_{K} x$, such that

$$
\left\|x-P_{K} x\right\| \leq\|x-y\| \quad \text { for all } y \in K .
$$

The mapping $P_{K}$ is called the metric projection from $H$ onto $K$. It is well known that $P_{K}$ has the following properties:

(i) $\left\langle x-y, P_{K} x-P_{K} y\right\rangle \geq\left\|P_{K} x-P_{K} y\right\|^{2}$ for every $x, y \in H$.

(ii) For $x \in H$ and $z \in K, z=P_{K}(x) \Leftrightarrow\langle x-z, z-y\rangle \geq 0$ for all $y \in K$.

(iii) For $x \in H$ and $y \in K$,

$$
\left\|y-P_{K}(x)\right\|^{2}+\left\|x-P_{K}(x)\right\|^{2} \leq\|x-y\|^{2} .
$$

Let $H_{1}$ and $H_{2}$ be two Hilbert spaces. Let $A: H_{1} \rightarrow H_{2}$ and $A^{*}: H_{2} \rightarrow H_{1}$ be two bounded linear operators. $A^{*}$ is called the adjoint operator (or adjoint) of $A$ if

$$
\langle A z, w\rangle=\left\langle z, A^{*} w\right\rangle \quad \text { for all } z \in H_{1} \text { and } w \in H_{2} \text {. }
$$

It is known that the adjoint operator of a bounded linear operator on a Hilbert space always exists and is bounded linear and unique. Moreover, it is not hard to show that if $A^{*}$ is an

○2014 He and Du; licensee Springer. This is an Open Access article distributed under the terms of the Creative Commons Attribution License (http://creativecommons.org/licenses/by/2.0), which permits unrestricted use, distribution, and reproduction in any medium, provided the original work is properly cited. 
adjoint operator of $A$, then $\|A\|=\left\|A^{*}\right\|$. The symbols $\mathbb{N}$ and $\mathbb{R}$ are used to denote the sets of positive integers and real numbers, respectively.

Example 1.1 ([1]) Let $H_{2}=\mathbb{R}$ with the standard norm $|\cdot|$ and $H_{1}=\mathbb{R}^{2}$ with the norm $\|\alpha\|=\left(a_{1}^{2}+a_{2}^{2}\right)^{\frac{1}{2}}$ for some $\alpha=\left(a_{1}, a_{2}\right) \in \mathbb{R}^{2} .\langle x, y\rangle=x y$ denotes the inner product of $H_{2}$ for some $x, y \in H_{2}$, and $\langle\alpha, \beta\rangle=\sum_{i=1}^{2} a_{i} b_{i}$ denotes the inner product of $H_{1}$ for some $\alpha=$ $\left(a_{1}, a_{2}\right), \beta=\left(b_{1}, b_{2}\right) \in H_{1}$. Let $A \alpha=a_{2}-a_{1}$ for $\alpha=\left(a_{1}, a_{2}\right) \in H_{1}$ and $B x=(-x, x)$ for $x \in H_{2}$, then $B$ is an adjoint operator of $A$.

Example 1.2 ([1]) Let $H_{1}=\mathbb{R}^{2}$ with the norm $\|\alpha\|=\left(a_{1}^{2}+a_{2}^{2}\right)^{\frac{1}{2}}$ for some $\alpha=\left(a_{1}, a_{2}\right) \in \mathbb{R}^{2}$ and $H_{2}=\mathbb{R}^{3}$ with the norm $\|\gamma\|=\left(c_{1}^{2}+c_{2}^{2}+c_{3}^{2}\right)^{\frac{1}{2}}$ for some $\gamma=\left(c_{1}, c_{2}, c_{3}\right) \in \mathbb{R}^{3}$. Let $\langle\alpha, \beta\rangle=$ $\sum_{i=1}^{2} a_{i} b_{i}$ and $\langle\gamma, \eta\rangle=\sum_{i=1}^{3} c_{i} d_{i}$ denote the inner product of $H_{1}$ and $H_{2}$, respectively, where $\alpha=\left(a_{1}, a_{2}\right), \beta=\left(b_{1}, b_{2}\right) \in H_{1}, \gamma=\left(c_{1}, c_{2}, c_{3}\right), \eta=\left(d_{1}, d_{2}, d_{3}\right) \in H_{2}$. Let $A \alpha=\left(a_{2}, a_{1}, a_{1}-a_{2}\right)$ for $\alpha=\left(a_{1}, a_{2}\right) \in H_{1}$ and $B \gamma=\left(c_{2}+c_{3}, c_{1}-c_{3}\right)$ for $\gamma=\left(c_{1}, c_{2}, c_{3}\right) \in H_{2}$. Obviously, $B$ is an adjoint operator of $A$.

Let $f$ be a bi-function from $C \times C$ to $\mathbb{R}$. The classical equilibrium problem (EP, for short) is defined as follows.

(EP) Find $p \in C$ such that $f(p, y) \geq 0, \forall y \in C$.

The set of such solutions is denoted by $\operatorname{EP}(f)$, that is, $\operatorname{EP}(f)=\{u \in C: f(u, v) \geq 0, \forall v \in$ $C$ \}. In fact, equilibrium problem has an important relationship with variational inequality problem. For example, let $T: C \rightarrow H$ be a nonlinear mapping satisfying $\langle T x, y-x\rangle \geq 0$ for all $x, y \in C$. Then $x \in \operatorname{EP}(f)$ if and only if $x \in C$ is a solution of the variational inequality $\langle T x, y-x\rangle \geq 0$ for all $y \in C$. It is known that the EP is an important mathematical model for nonlinear analysis and applied sciences which is generalized to many new mathematical models and includes many important problems arising in physics, engineering, science optimization, economics, network, game theory, complementary problems, variational inequalities problems, saddle point problems, fixed point problems and others; for details, one can refer to [2-8] and references therein. Many authors have proposed some useful methods to solve the EP; see, for instance, [2-5, 9-17] and references therein.

Recent investigations and developments in equilibrium theory as well as optimization theory have been applied to connect fundamental sciences with the real world. According to our experience, useful methods of real world problems often need to be used to solve several problems arising in different spaces. In view of this, recent studies focus on split problems which are more closed in the real world applications; see, for instance, [1, 1824] and the references therein. Recently, He [1] considered the following split equilibrium problem. Let $H_{1}$ and $H_{2}$ be two real Hilbert spaces. Let $C$ be a closed convex subset of $H_{1}$ and $K$ be a closed convex subset of $H_{2}$. Let $f: C \times C \rightarrow \mathbb{R}$ and $g: K \times K \rightarrow \mathbb{R}$ be two bifunctions, and $A: H_{1} \rightarrow H_{2}$ be a bounded linear operator. The split equilibrium problem (SEP, in short) is defined as follows:

(SEP) Find $p \in C$ such that $f(p, y) \geq 0, \forall y \in C$, and $u:=A p$ satisfying $g(u, v) \geq 0, \forall v \in K$.

In [1], the author established weak convergence algorithms and strong convergence algorithms for SEP (see [1] for more details). 
Motivated and inspired by the works mentioned above, in this paper we shall introduce and investigate the following new problem. Let $H_{1}, H_{2}$ and $H_{3}$ be three real Hilbert spaces. Let $C$ be a closed convex subset of $H_{1}, Q$ be a closed convex subset of $H_{2}$ and $K$ be a closed convex subset of $H_{3}$. Let $f: C \times C \rightarrow \mathbb{R}, g: Q \times Q \rightarrow \mathbb{R}$ and $h: K \times K \rightarrow \mathbb{R}$ be three bi-functions. Let $A: H_{1} \rightarrow H_{3}$ and $B: H_{2} \rightarrow H_{3}$ be two bounded linear operators with theirs adjoint operators $A^{*}$ and $B^{*}$, respectively. The mathematical model about bilevel split equilibrium problem (BSEP, in short) is defined as follows:

(BSEP) Find $p \in C$ and $q \in Q$ such that

(i) $f(p, x) \geq 0$ and $g(q, y) \geq 0$ for all $x \in C$ and $y \in Q$;

(ii) $A p=B q:=u$;

(iii) $h(u, z) \geq 0$ for all $z \in K$.

In fact, BSEP can be regarded as a new development in the field of equilibrium problems and contains several important problems as special cases. It was profoundly believed that BSEP will motivate and inspire further scientific activities in the fields of equilibrium problems, optimization problems, game problems, complementary problems, variational inequalities problems, fixed point problems and their applications.

Example A Let $H_{1}, H_{2}$ and $H_{3}$ be three real Hilbert spaces. Let $C \subset H_{1}, Q \subset H_{2}$ and $K \subset H_{3}$ be three closed convex sets. Let $f^{*}: C \rightarrow \mathbb{R}, g^{*}: Q \rightarrow \mathbb{R}$ and $h^{*}: K \rightarrow \mathbb{R}$ be three convex functions. Let $A: H_{1} \rightarrow H_{3}$ and $B: H_{2} \rightarrow H_{3}$ be two bounded linear operators with their adjoint operators $A^{*}$ and $B^{*}$, respectively. Let

$$
\begin{array}{ll}
f(x, \alpha)=f^{*}(x)-f^{*}(\alpha) & \text { for } x, \alpha \in C, \\
g(y, \beta)=g^{*}(y)-g^{*}(\beta) & \text { for } y, \beta \in Q,
\end{array}
$$

and

$$
h(z, \eta)=h^{*}(z)-h^{*}(\eta) \quad \text { for } z, \eta \in K .
$$

Then BSEP reduces the bilevel convex optimization problem (BCOP):

(BCOP) Find $p \in C$ and $q \in Q$ such that $u:=A p=B q \in K, f^{*}(x) \geq f^{*}(p), g^{*}(y) \geq g^{*}(q)$ and $h^{*}(z) \geq h^{*}(u)$ for all $x \in C, y \in Q$ and $z \in K$.

Example B Let $H_{1}, H_{2}$ and $H_{3}$ be three real Hilbert spaces. Let $C \subset H_{1}, Q \subset H_{2}$ and $K \subset$ $H_{3}$ be three closed convex sets. Let $T: C \rightarrow H_{1}, S: Q \rightarrow H_{2}$ and $G: K \rightarrow H_{3}$ be three nonlinear operators. Let $A: H_{1} \rightarrow H_{3}$ and $B: H_{2} \rightarrow H_{3}$ be two bounded linear operators with their adjoint operators $A^{*}$ and $B^{*}$, respectively. If $f(p, x)=\langle T p, x-p\rangle, g(q, y)=\langle S q, y-$ $q\rangle$ and $h(u, z)=\langle T u, z-u\rangle$, then BSEP reduces to the bilevel split variational inequality problem (BSVI):

(BSVI) Find $p \in C$ and $q \in Q$ such that $u:=A p=B q \in K$ satisfying $\langle T p, x-p\rangle \geq 0,\langle S q, y-$ $q\rangle \geq 0$ and $\langle T u, z-u\rangle \geq 0$ for all $x \in C, y \in Q$ and $z \in K$. 
Example C Let $H_{1}$ and $H_{2}$ be two real Hilbert spaces and $B: H_{1} \rightarrow H_{2}$ be a bounded linear operator with its adjoint operator $B^{*}$. Let $C \subset H_{1}, Q \subset H_{1}$ and $K \subset H_{2}$ be three closed convex sets. If $H_{1}=H_{2}$ and $A=B$, then BSEP reduces to the following split equilibrium problem (1) $\left(\mathrm{SEP}^{(1)}\right)$ :

$\left(\mathrm{SEP}^{(1)}\right)$ Find $p \in C$ and $q \in Q$ such that $u:=B p=B q \in K$ satisfying $f(p, x) \geq 0, g(q, y) \geq 0$ and $h(u, z) \geq 0$ for all $x \in C, y \in Q$ and $z \in K$.

Example D Let $H_{1}$ and $H_{2}$ be two real Hilbert spaces and $A: H_{1} \rightarrow H_{2}$ be a bounded linear operator with its adjoint operator $A^{*}$. Let $C \subset H_{1}, Q \subset H_{2}$ and $K \subset H_{2}$ be three closed convex sets with $Q \cap K \neq \emptyset$. If $H_{2}=H_{3}$ and $B=I$ (identity operator), then BSEP reduces to the following split equilibrium problem $(2)\left(\operatorname{SEP}^{(2)}\right)$ :

$\left(\operatorname{SEP}^{(2)}\right)$ Find $p \in C$ such that $u:=A p \in Q \cap K$ satisfying $f(p, x) \geq 0, g(u, y) \geq 0$ and $h(u, z) \geq 0$ for each $x \in C, y \in Q$ and $z \in K$.

Especially, if $g(p, y) \equiv 0$ for all $p, y \in Q$, then $\left(\operatorname{SEP}^{(1)}\right)$ reduces to finding $p \in C$ such that $u:=A p \in K$ satisfying $f(p, x) \geq 0$ and $h(u, z) \geq 0$ for all $x \in C$ and $z \in K$, which was studied in $[1]$.

Example E Let $H_{1}$ and $H_{2}$ be two real Hilbert spaces and $B: H_{1} \rightarrow H_{2}$ be a bounded linear operator with its adjoint operator $A^{*}$. Let $C \subset H_{1}, Q \subset H_{1}$ and $K \subset H_{2}$ be three closed convex sets with $C \cap Q \neq \emptyset$. If $H_{1}=H_{2}$ and $A=I$ (identity operator), then BSEP reduces to the following split equilibrium problems $(3)\left(\mathrm{SEP}^{(3)}\right)$ :

$\left(\mathrm{SEP}^{(3)}\right)$ Find $p \in C \cap Q$ such that $u:=B p \in K$ satisfying $f(p, x) \geq 0, g(p, y) \geq 0$ and $h(u, z) \geq 0$ for all $x \in C, y \in Q$ and $z \in K$.

Example F In Example A, if $H_{1}=H_{2}=H_{3}:=H, C=Q=K \subset H$ and $A=B=I$ (identity operator), then BSEP reduces to the common solution of equilibrium problems (CEP):

(CEP) Find $p \in C$ such that $f(p, x) \geq 0, g(p, y) \geq 0$ and $h(p, z) \geq 0$ for each $x, y, z \in C$.

The paper is divided into four sections. In Sections 1 and 2, we first introduce and investigate a bilevel split equilibrium problem (BSEP) and then provide some new feasible iterative algorithms for BSEP and establish strong convergence theorems for these iterative algorithms in different spaces. In Section 3, we give the proof of the main result Theorem 2.1 in detail. Finally, an example illustrating Theorem 2.1 is given in Section 4 .

\section{Feasible iterative algorithms for BSEP and their strong convergence theorems}

In 1994, Blum and Oettli [2] established the following important existence theorem which plays a key role in solving equilibrium problems, variational inequality problems and optimization problems.

Lemma 2.1 (Blum and Oettli [2]) Let $K$ be a nonempty closed convex subset of $H$ and $F$ be a bi-function of $K \times K$ into $\mathbb{R}$ satisfying the following conditions.

(A1) $F(x, x)=0$ for all $x \in K$; 
(A2) $F$ is monotone, that is, $F(x, y)+F(y, x) \leq 0$ for all $x, y \in K$;

(A3) for each $x, y, z \in K$,

$$
\limsup _{t \rightarrow 0^{+}} F(t z+(1-t) x, y) \leq F(x, y)
$$

(A4) for each $x \in K, y \mapsto F(x, y)$ is convex and lower semi-continuous.

Let $r>0$ and $x \in H$. Then there exists $z \in K$ such that

$$
F(z, y)+\frac{1}{r}\langle y-z, z-x\rangle \geq 0 \quad \text { for all } y \in K .
$$

In this paper, we first introduce a new iterative algorithm for BSEP and establish a strong convergence theorem for this iterative algorithm. Here, the space $H_{1} \times H_{2}$ denotes the product space of two real Hilbert spaces $H_{1}$ and $H_{2}$, which is endowed with the usual linear operation and norm, namely, for $(x, y),(\bar{x}, \bar{y}) \in H_{1} \times H_{2}$ and $a, b \in \mathbb{R}$,

$$
a(x, y)+b(\bar{x}, \bar{y})=(a x+b \bar{x}, a y+b \bar{y})
$$

and

$$
\|(x, y)\|=\|x\|+\|y\| .
$$

Theorem 2.1 Let $H_{1}, H_{2}$ and $H_{3}$ be three real Hilbert spaces. Let $C$ be a closed convex subset of $H_{1}, Q$ be a closed convex subset of $H_{2}$ and $K$ be a closed convex subset of $H_{3}$. Let $f: C \times C \rightarrow \mathbb{R}, g: Q \times Q \rightarrow \mathbb{R}$ and $h: K \times K \rightarrow \mathbb{R}$ be three bi-functions. $A: H_{1} \rightarrow H_{3}$ and $B: H_{2} \rightarrow H_{3}$ are two bounded linear operators with their adjoint operators $A^{*}$ and $B^{*}$, respectively. Suppose that all the bi-functions $f, g$ and $h$ satisfy conditions (A1)-(A4). Let $x_{1} \in C, y_{1} \in Q, C_{1}=C, Q_{1}=Q,\left\{x_{n}\right\},\left\{y_{n}\right\},\left\{u_{n}\right\},\left\{v_{n}\right\}$ and $\left\{w_{n}\right\}$ be sequences generated by

$$
\left\{\begin{array}{l}
u_{n}=T_{r_{n}}^{f} x_{n}, \quad v_{n}=T_{r_{n}}^{g} y_{n}, \quad w_{n}=T_{r_{n}}^{h}\left(\frac{1}{2} A u_{n}+\frac{1}{2} B v_{n}\right), \\
l_{n}=P_{C}\left(u_{n}-\xi A^{*}\left(A u_{n}-w_{n}\right)\right), \quad k_{n}=P_{Q}\left(v_{n}-\xi B^{*}\left(B v_{n}-w_{n}\right)\right), \\
C_{n+1} \times Q_{n+1}=\left\{(x, y) \in C_{n} \times Q_{n}:\left\|l_{n}-x\right\|^{2}+\left\|k_{n}-y\right\|^{2}\right. \\
\left.\quad \leq\left\|u_{n}-x\right\|^{2}+\left\|v_{n}-y\right\|^{2} \leq\left\|x_{n}-x\right\|^{2}+\left\|y_{n}-y\right\|^{2}\right\}, \\
x_{n+1}=P_{C_{n+1}}\left(x_{1}\right), \quad \\
y_{n+1}=P_{Q_{n+1}}\left(y_{1}\right), \quad \forall n \in \mathbb{N},
\end{array}\right.
$$

where $\xi \in\left(0, \min \left(\frac{1}{\|A\|^{2}}, \frac{1}{\|B\|^{2}}\right)\right)$ and $\left\{r_{n}\right\} \subset(0,+\infty)$ with $\liminf _{n \rightarrow+\infty} r_{n}>0, P_{C}$ and $P_{Q}$ are two projection operators from $H_{1}$ into $C$ and from $H_{2}$ into $Q$, respectively. Suppose that

$$
\Omega=\{(p, q) \in \mathrm{EP}(f) \times \mathrm{EP}(g): A p=B q \in \mathrm{EP}(h)\} \neq \emptyset .
$$

Then there exists $(p, q) \in \Omega$ such that

(a) $\left(x_{n}, y_{n}\right) \rightarrow(p, q)$ as $n \rightarrow \infty$;

(b) $\left(u_{n}, v_{n}\right) \rightarrow(p, q)$ as $n \rightarrow \infty$;

(c) $w_{n} \rightarrow w^{*}:=A p=B q \in \mathrm{EP}(h)$ as $n \rightarrow \infty$. 
The following conclusion is immediate from Theorem 2.1 by putting $A=B$.

Corollary 2.1 Let $H_{1}$ and $H_{2}$ be two real Hilbert spaces. Let $C$ and $Q$ be two closed convex subsets of $H_{1}$ and $K$ be a closed convex subset of $H_{2}$. Letf $: C \times C \rightarrow \mathbb{R}, g: Q \times Q \rightarrow \mathbb{R}$ and $h$ : $K \times K \rightarrow \mathbb{R}$ be three bi-functions. $B: H_{1} \rightarrow H_{2}$ is a bounded linear operator with its adjoint operator $B^{*}$. Suppose that all the bi-functions $f, g$ and h satisfy conditions (A1)-(A4). Let $x_{1} \in C, y_{1} \in Q, C_{1}=C, Q_{1}=Q,\left\{x_{n}\right\},\left\{y_{n}\right\},\left\{u_{n}\right\},\left\{v_{n}\right\}$ and $\left\{w_{n}\right\}$ be sequences generated by

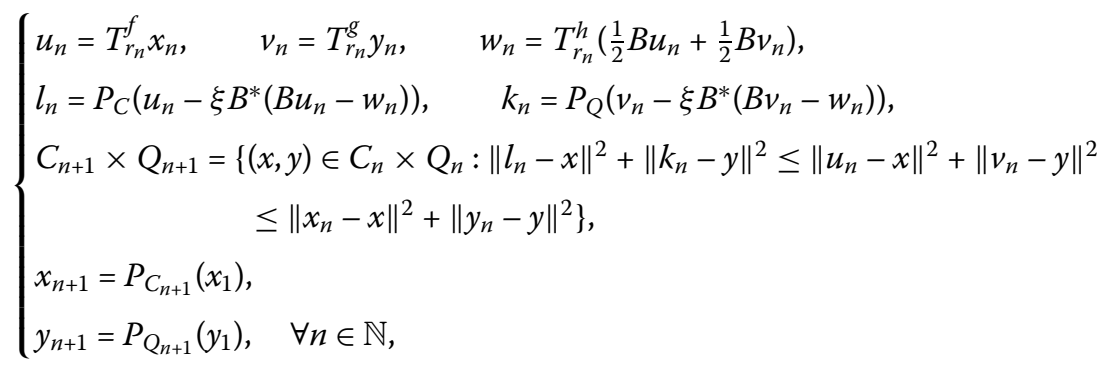

where $\xi \in\left(0, \frac{1}{\|B\|^{2}}\right)$ and $\left\{r_{n}\right\} \subset(0,+\infty)$ with $\liminf _{n \rightarrow+\infty} r_{n}>0, P_{C}$ and $P_{Q}$ are two projection operators from $H_{1}$ into $C$ and from $H_{1}$ into $Q$, respectively. Suppose that

$$
\Omega=\{(p, q) \in \mathrm{EP}(f) \times \mathrm{EP}(g): B p=B q \in \mathrm{EP}(h)\} \neq \emptyset .
$$

Then there exists $(p, q) \in \Omega$ such that

(a) $\left(x_{n}, y_{n}\right) \rightarrow(p, q)$ as $n \rightarrow \infty$;

(b) $\left(u_{n}, v_{n}\right) \rightarrow(p, q)$ as $n \rightarrow \infty$;

(c) $w_{n} \rightarrow w^{*}:=B p=B q \in \mathrm{EP}(h)$ as $n \rightarrow \infty$.

If $H_{2}=H_{3}$ and $B=I$, then Theorem 2.1 reduces to the following corollary.

Corollary 2.2 Let $H_{1}$ and $H_{2}$ be two real Hilbert spaces. Let $C \subset H_{1}$ and $Q, K \subset H_{2}$ be three closed convex sets. Let $f: C \times C \rightarrow \mathbb{R}, g: Q \times Q \rightarrow \mathbb{R}$ and $h: K \times K \rightarrow \mathbb{R}$ be three bifunctions. $A: H_{1} \rightarrow H_{2}$ is a bounded linear operator with its adjoint operator $A^{*}$. Suppose that all the bi-functions $f, g$ and $h$ satisfy conditions (A1)-(A4). Let $x_{1} \in C, y_{1} \in Q, C_{1}=C$, $Q_{1}=Q,\left\{x_{n}\right\},\left\{y_{n}\right\},\left\{u_{n}\right\},\left\{v_{n}\right\}$ and $\left\{w_{n}\right\}$ be sequences generated by

$$
\left\{\begin{array}{l}
u_{n}=T_{r_{n}}^{f} x_{n}, \quad v_{n}=T_{r_{n}}^{g} y_{n}, \quad w_{n}=T_{r_{n}}^{h}\left(\frac{1}{2} A u_{n}+\frac{1}{2} v_{n}\right), \\
l_{n}=P_{C}\left(u_{n}-\xi A^{*}\left(A u_{n}-w_{n}\right)\right), \quad k_{n}=v_{n}-\xi\left(v_{n}-w_{n}\right), \\
C_{n+1} \times Q_{n+1}=\left\{(x, y) \in C_{n} \times Q_{n}:\left\|l_{n}-x\right\|^{2}+\left\|k_{n}-y\right\|^{2} \leq\left\|u_{n}-x\right\|^{2}+\left\|v_{n}-y\right\|^{2}\right. \\
\left.\quad \leq\left\|x_{n}-x\right\|^{2}+\left\|y_{n}-y\right\|^{2}\right\}, \\
x_{n+1}=P_{C_{n+1}}\left(x_{1}\right), \\
y_{n+1}=P_{Q_{n+1}}\left(y_{1}\right), \quad \forall n \in \mathbb{N},
\end{array}\right.
$$

where $\xi \in\left(0, \min \left\{1, \frac{1}{\|A\|^{2}}\right\}\right)$ and $\left\{r_{n}\right\} \subset(0,+\infty)$ with $\liminf _{n \rightarrow+\infty} r_{n}>0, P_{C}$ and $P_{Q}$ are two projection operators from $\mathrm{H}_{1}$ into $C$ and from $\mathrm{H}_{2}$ into $Q$, respectively. Suppose that

$$
\Omega=\{p \in \operatorname{EP}(f): A p \in \operatorname{EP}(g) \cap \operatorname{EP}(h)\} \neq \emptyset .
$$


Then there exists $p \in \Omega$ such that

(a) $x_{n} \rightarrow p$ as $n \rightarrow \infty$;

(b) $u_{n} \rightarrow p$ as $n \rightarrow \infty$;

(c) $v_{n}, y_{n}, w_{n} \rightarrow w^{*}:=$ Ap as $n \rightarrow \infty$.

If $H_{1}=H_{2}$ and $A=I$, then Theorem 2.1 reduces to the following corollary.

Corollary 2.3 Let $H_{1}$ and $H_{2}$ be two real Hilbert spaces. Let $C, Q \subset H_{1}$ and $K \subset H_{2}$ be three closed convex sets. Let $f: C \times C \rightarrow \mathbb{R}, g: Q \times Q \rightarrow \mathbb{R}$ and $h: K \times K \rightarrow \mathbb{R}$ be three bifunctions. $B: H_{1} \rightarrow H_{2}$ is a bounded linear operator with its adjoint operator $B^{*}$. Suppose that all the bi-functions $f, g$ and $h$ satisfy conditions (A1)-(A4). Let $x_{1} \in C, y_{1} \in Q, C_{1}=C$, $Q_{1}=Q,\left\{x_{n}\right\},\left\{y_{n}\right\},\left\{u_{n}\right\},\left\{v_{n}\right\}$ and $\left\{w_{n}\right\}$ be sequences generated by

$$
\left\{\begin{array}{l}
u_{n}=T_{r_{n}}^{f} x_{n}, \quad v_{n}=T_{r_{n}}^{g} y_{n}, \quad w_{n}=T_{r_{n}}^{h}\left(\frac{1}{2} u_{n}+\frac{1}{2} B v_{n}\right), \\
l_{n}=u_{n}-\xi\left(u_{n}-w_{n}\right), \quad k_{n}=P_{q}\left(v_{n}-\xi B^{*}\left(B v_{n}-w_{n}\right)\right), \\
\quad \begin{array}{l}
C_{n+1} \times Q_{n+1}=\left\{(x, y) \in C_{n} \times Q_{n}:\left\|l_{n}-x\right\|^{2}+\left\|k_{n}-y\right\|^{2} \leq\left\|u_{n}-x\right\|^{2}+\left\|v_{n}-y\right\|^{2}\right. \\
\left.\quad \leq\left\|x_{n}-x\right\|^{2}+\left\|y_{n}-y\right\|^{2}\right\},
\end{array} \\
\begin{array}{l}
x_{n+1}=P_{C_{n+1}}\left(x_{1}\right), \\
y_{n+1}=P_{Q_{n+1}}\left(y_{1}\right), \quad \forall n \in \mathbb{N},
\end{array}
\end{array}\right.
$$

where $\xi \in\left(0, \min \left\{1, \frac{1}{\|B\|^{2}}\right\}\right)$ and $\left\{r_{n}\right\} \subset(0,+\infty)$ with $\liminf _{n \rightarrow+\infty} r_{n}>0, P_{C}$ and $P_{Q}$ are two projection operators from $\mathrm{H}_{1}$ into $C$ and from $\mathrm{H}_{2}$ into $Q$, respectively. Suppose that

$$
\Omega=\{p \in \mathrm{EP}(f) \cap \mathrm{EP}(g): A p \in \mathrm{EP}(h)\} \neq \emptyset .
$$

Then there exists $p \in \Omega$ such that

(a) $x_{n}, u_{n} \rightarrow p$ as $n \rightarrow \infty$;

(b) $y_{n}, v_{n} \rightarrow p$ as $n \rightarrow \infty$;

(c) $w_{n} \rightarrow w^{*}:=A p$ as $n \rightarrow \infty$.

Putting $A=B=I$ (identical operator), $H_{1}=H_{2}=H_{3}=H$ and $C=Q=K$, then we have the following result.

Corollary 2.4 Let $H$ be a real Hilbert space. Let $C$ be a closed convex subset of H. Let $f, g, h: C \times C \rightarrow \mathbb{R}$ be three bi-functions. Suppose that all the bi-functions $f, g$ and $h$ satisfy conditions (A1)-(A4). Let $x_{1}, y_{1} \in C, C_{1}=C,\left\{x_{n}\right\},\left\{y_{n}\right\},\left\{u_{n}\right\},\left\{v_{n}\right\}$ and $\left\{w_{n}\right\}$ be sequences generated by

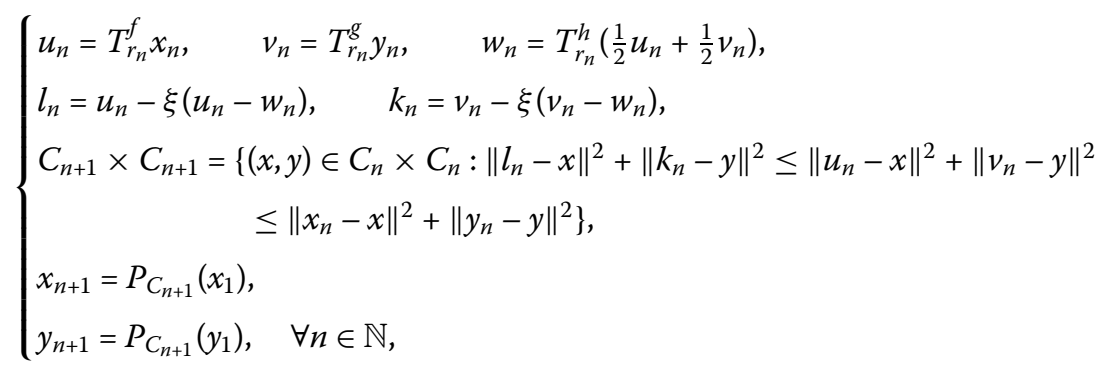


where $\xi \in(0,1)$ and $\left\{r_{n}\right\} \subset(0,+\infty)$ with $\liminf _{n \rightarrow+\infty} r_{n}>0, P_{C}$ is a projection operator from $H$ into C. Suppose that

$$
\Omega=\{(p, q) \in \mathrm{EP}(f) \times \mathrm{EP}(g): p=q \in \mathrm{EP}(h)\} \neq \emptyset .
$$

Then there exists $(p, q) \in \Omega$ such that

(a) $\left(x_{n}, y_{n}\right) \rightarrow(p, q)$ as $n \rightarrow \infty$;

(b) $\left(u_{n}, v_{n}\right) \rightarrow(p, q)$ as $n \rightarrow \infty$;

(c) $w_{n} \rightarrow w^{*}:=p=q \in \mathrm{EP}(h)$ as $n \rightarrow \infty$.

Remark 2.1 In Corollary 2.2, it is obvious that

$$
\Omega=\{(p, q) \in \mathrm{EP}(f) \times \operatorname{EP}(g): p=q \in \operatorname{EP}(h)\} \neq \emptyset
$$

implies

$$
\Omega=\{p \in \operatorname{EP}(f) \cap \operatorname{EP}(g) \cap \operatorname{EP}(h)\} \neq \emptyset .
$$

Hence, the problem studied in Corollary 2.4 is still the study of a common solution of three equilibrium problems in essence.

If $C, Q, K$ are linear subspaces of a real Hilbert space, then we have the following corollaries from Theorem 2.1 and Corollary 2.1.

Corollary 2.5 Let $H_{1}, H_{2}$ and $H_{3}$ be three real Hilbert spaces. Let $C \subset H_{1}, Q \subset H_{2}$ and $K \subset H_{3}$ be three linear subspaces. Let $f: C \times C \rightarrow \mathbb{R}, g: Q \times Q \rightarrow \mathbb{R}$ and $h: K \times K \rightarrow \mathbb{R}$ be three bi-functions. $A: H_{1} \rightarrow H_{3}$ and $B: H_{2} \rightarrow H_{3}$ are two bounded linear operators with their adjoint operators $A^{*}$ and $B^{*}$, respectively. Suppose that all the bi-functions $f, g$ and $h$ satisfy conditions (A1)-(A4). Let $x_{1} \in C, y_{1} \in Q, C_{1}=C, Q_{1}=Q,\left\{x_{n}\right\},\left\{y_{n}\right\},\left\{u_{n}\right\},\left\{v_{n}\right\}$ and $\left\{w_{n}\right\}$ be sequences generated by

$$
\left\{\begin{array}{l}
u_{n}=T_{r_{n}}^{f} x_{n}, \quad v_{n}=T_{r_{n}}^{g} y_{n}, \quad w_{n}=T_{r_{n}}^{h}\left(\frac{1}{2} A u_{n}+\frac{1}{2} B v_{n}\right), \\
l_{n}=u_{n}-\xi A^{*}\left(A u_{n}-w_{n}\right), \quad k_{n}=v_{n}-\xi B^{*}\left(B v_{n}-w_{n}\right), \\
\quad \begin{array}{l}
C_{n+1} \times Q_{n+1}=\left\{(x, y) \in C_{n} \times Q_{n}:\left\|l_{n}-x\right\|^{2}+\left\|k_{n}-y\right\|^{2} \leq\left\|u_{n}-x\right\|^{2}+\left\|v_{n}-y\right\|^{2}\right. \\
\left.\quad \leq\left\|x_{n}-x\right\|^{2}+\left\|y_{n}-y\right\|^{2}\right\}, \\
x_{n+1}=P_{C_{n+1}}\left(x_{1}\right),
\end{array} \\
y_{n+1}=P_{Q_{n+1}}\left(y_{1}\right), \quad \forall n \in \mathbb{N},
\end{array}\right.
$$

where $\xi \in\left(0, \min \left(\frac{1}{\|A\|^{2}}, \frac{1}{\|B\|^{2}}\right)\right)$ and $\left\{r_{n}\right\} \subset(0,+\infty)$ with $\liminf _{n \rightarrow+\infty} r_{n}>0, P_{C}$ and $P_{Q}$ are two projection operators from $H_{1}$ into $C$ and from $H_{2}$ into $Q$, respectively. Suppose that

$$
\Omega=\{(p, q) \in \mathrm{EP}(f) \times \mathrm{EP}(g): A p=B q \in \operatorname{EP}(h)\} \neq \emptyset .
$$

Then there exists $(p, q) \in \Omega$ such that

(a) $\left(x_{n}, y_{n}\right) \rightarrow(p, q)$ as $n \rightarrow \infty$; 
(b) $\left(u_{n}, v_{n}\right) \rightarrow(p, q)$ as $n \rightarrow \infty$;

(c) $w_{n} \rightarrow w^{*}:=A p=B q \in \mathrm{EP}(h)$ as $n \rightarrow \infty$.

Corollary 2.6 Let $H_{1}$ and $H_{2}$ be two real Hilbert spaces. Let $C \subset H_{1}, Q \subset H_{1}$ and $K \subset H_{2}$ be three linear subspaces. Let $f: C \times C \rightarrow \mathbb{R}, g: Q \times Q \rightarrow \mathbb{R}$ and $h: K \times K \rightarrow \mathbb{R}$ be three bifunctions. $B: H_{1} \rightarrow H_{2}$ is a bounded linear operator with its adjoint operator $B^{*}$. Suppose that all the bi-functions $f, g$ and $h$ satisfy conditions (A1)-(A4). Let $x_{1} \in C, y_{1} \in Q, C_{1}=C$, $Q_{1}=Q,\left\{x_{n}\right\},\left\{y_{n}\right\},\left\{u_{n}\right\},\left\{v_{n}\right\}$ and $\left\{w_{n}\right\}$ be sequences generated by

$$
\left\{\begin{array}{l}
u_{n}=T_{r_{n}}^{f} x_{n}, \quad v_{n}=T_{r_{n}}^{g} y_{n}, \quad w_{n}=T_{r_{n}}^{h}\left(\frac{1}{2} B u_{n}+\frac{1}{2} B v_{n}\right), \\
\quad \begin{array}{l}
l_{n}=u_{n}-\xi B^{*}\left(B u_{n}-w_{n}\right), \quad k_{n}=v_{n}-\xi B^{*}\left(B v_{n}-w_{n}\right), \\
C_{n+1} \times Q_{n+1}=\left\{(x, y) \in C_{n} \times Q_{n}:\left\|l_{n}-x\right\|^{2}+\left\|k_{n}-y\right\|^{2} \leq\left\|u_{n}-x\right\|^{2}+\left\|v_{n}-y\right\|^{2}\right. \\
\left.\quad \leq\left\|x_{n}-x\right\|^{2}+\left\|y_{n}-y\right\|^{2}\right\}, \\
x_{n+1}=P_{C_{n+1}}\left(x_{1}\right),
\end{array} \\
y_{n+1}=P_{Q_{n+1}}\left(y_{1}\right), \quad \forall n \in \mathbb{N},
\end{array}\right.
$$

where $\xi \in\left(0, \frac{1}{\|B\|^{2}}\right)$ and $\left\{r_{n}\right\} \subset(0,+\infty)$ with $\liminf _{n \rightarrow+\infty} r_{n}>0, P_{C}$ and $P_{Q}$ are two projection operators from $H_{1}$ into $C$ and from $H_{1}$ into $Q$, respectively. Suppose that

$$
\Omega=\{(p, q) \in \mathrm{EP}(f) \times \mathrm{EP}(g): B p=B q \in \mathrm{EP}(h)\} \neq \emptyset .
$$

Then there exists $(p, q) \in \Omega$ such that

(a) $\left(x_{n}, y_{n}\right) \rightarrow(p, q)$ as $n \rightarrow \infty$;

(b) $\left(u_{n}, v_{n}\right) \rightarrow(p, q)$ as $n \rightarrow \infty$;

(c) $w_{n} \rightarrow w^{*}:=B p=B q \in \mathrm{EP}(h)$ as $n \rightarrow \infty$.

Remark 2.2 In fact, the problem studied by Corollaries 2.1-2.3 and Corollary 2.6 is (SEP).

In order to prove Theorem 2.1, we need the following crucial known results.

Lemma 2.2 (see [10]) Let $K$ be a nonempty closed convex subset of $H$, and let $F$ be a bifunction of $K \times K$ into $\mathbb{R}$ satisfying (A1)-(A4). For $r>0$, define a mapping $T_{r}^{F}: H \rightarrow K$ as follows:

$$
T_{r}^{F}(x)=\left\{z \in K: F(z, y)+\frac{1}{r}\langle y-z, z-x\rangle \geq 0, \forall y \in K\right\}
$$

for all $x \in H$. Then the following hold:

(i) $T_{r}^{F}$ is single-valued and $\mathcal{F}\left(T_{r}^{F}\right)=\mathrm{EP}(F)$ for $\forall r>0$ and $\mathrm{EP}(F)$ is closed and convex;

(ii) $T_{r}^{F}$ is firmly nonexpansive, that is, for any $x, y \in H$, $\left\|T_{r}^{F} x-T_{r}^{F} y\right\|^{2} \leq\left\langle T_{r}^{F} x-T_{r}^{F} y, x-y\right\rangle$.

Lemma 2.3 ([20]) Let $F_{r}^{F}$ be the same as in Lemma 2.2. If $\mathcal{F}\left(T_{r}^{F}\right)=\mathrm{EP}(F) \neq \emptyset$, then, for any $x \in H$ and $x^{*} \in \mathcal{F}\left(T_{r}^{F}\right),\left\|T_{r}^{F} x-x\right\|^{2} \leq\left\|x-x^{*}\right\|^{2}-\left\|T_{r}^{F} x-x^{*}\right\|^{2}$. 
Lemma 2.4 ([1,19]) Let the mapping $T_{r}^{F}$ be defined as in Lemma 2.2. Then, for $r, s>0$ and $x, y \in H$,

$$
\left\|T_{r}^{F}(x)-T_{s}^{F}(y)\right\| \leq\|x-y\|+\frac{|s-r|}{s}\left\|T_{s}^{F}(y)-y\right\| .
$$

In particular, $\left\|T_{r}^{F}(x)-T_{r}^{F}(y)\right\| \leq\|x-y\|$ for any $r>0$ and $x, y \in H$, that is, $T_{r}^{F}$ is nonexpansive for any $r>0$.

Lemma 2.5 (see, e.g., [25]) Let $H$ be a real Hilbert space. Then the following hold:

(a) $\|x-y\|^{2}=\|x\|^{2}+\|y\|^{2}-2\langle x, y\rangle$ for all $x, y \in H$;

(b) $\|\alpha x+(1-\alpha) y\|^{2}=\alpha\|x\|^{2}+(1-\alpha)\|y\|^{2}-\alpha(1-\alpha)\|x-y\|^{2}$ for all $x, y \in H$ and $\alpha \in[0,1]$.

\section{Proof of Theorem 2.1}

Applying Lemmas 2.1 and 2.2, we know that $\left\{u_{n}\right\},\left\{v_{n}\right\}$ and $\left\{w_{n}\right\}$ are all well defined. It is also easy to verify that $C_{n}, Q_{n}, C_{n} \times Q_{n}$ are closed convex sets for $n \in \mathbb{N}$.

Now, we claim $C_{n} \times Q_{n} \neq \emptyset$ for all $n \in \mathbb{N}$. Indeed, it suffices to prove that $\Omega \subset C_{n} \times Q_{n}$ for all $n \in \mathbb{N}$. Let $\left(x^{*}, y^{*}\right) \in \Omega$. Then $x^{*} \in \operatorname{EP}(f), y^{*} \in \operatorname{EP}(g)$ and

$$
w^{*}:=A x^{*}=B y^{*} \in \operatorname{EP}(h)
$$

Let $n \in \mathbb{N}$ be given. By Lemma 2.3, we have

$$
\begin{aligned}
& \left\|u_{n}-x^{*}\right\| \leq\left\|x_{n}-x^{*}\right\|, \quad\left\|v_{n}-y^{*}\right\| \leq\left\|y_{n}-y^{*}\right\|, \\
& \left\|w_{n}-w^{*}\right\| \leq\left\|\frac{A u_{n}+B v_{n}}{2}-w^{*}\right\|, \\
& \left\|w_{n}-w^{*}\right\|^{2} \leq \frac{1}{2}\left\|A u_{n}-w^{*}\right\|^{2}+\frac{1}{2}\left\|B v_{n}-w^{*}\right\|^{2} \quad \text { (by Lemma 2.5). }
\end{aligned}
$$

From (2.1), (3.1) and Lemma 2.5, we obtain

$$
\begin{aligned}
\left\|l_{n}-x^{*}\right\|^{2}= & \left\|P_{C}\left(u_{n}-\xi A^{*}\left(A u_{n}-w_{n}\right)\right)-x^{*}\right\|^{2} \leq\left\|u_{n}-x^{*}-\xi A^{*}\left(A u_{n}-w_{n}\right)\right\|^{2} \\
= & \left\|u_{n}-x^{*}\right\|^{2}+\left\|\xi A^{*}\left(A u_{n}-w_{n}\right)\right\|^{2}-2 \xi\left\langle u_{n}-x^{*}, A^{*}\left(A u_{n}-w_{n}\right)\right\rangle \\
= & \left\|u_{n}-x^{*}\right\|^{2}+\left\|\xi A^{*}\left(A u_{n}-w_{n}\right)\right\|^{2}-2 \xi\left\langle A u_{n}-A x^{*}, A u_{n}-w_{n}\right\rangle \\
= & \left\|u_{n}-x^{*}\right\|^{2}+\left\|\xi A^{*}\left(A u_{n}-w_{n}\right)\right\|^{2}-2 \xi\left\langle A u_{n}-w^{*}, A u_{n}-w_{n}\right\rangle \\
= & \left\|u_{n}-x^{*}\right\|^{2}+\left\|\xi A^{*}\left(A u_{n}-w_{n}\right)\right\|^{2}-\xi\left\|A u_{n}-w^{*}\right\|^{2} \\
& -\xi\left\|A u_{n}-w_{n}\right\|^{2}+\xi\left\|w_{n}-w^{*}\right\|^{2} \\
\leq & \left\|u_{n}-x^{*}\right\|^{2}-\xi\left(1-\xi\left\|A^{*}\right\|^{2}\right)\left\|A u_{n}-w_{n}\right\|^{2} \\
& -\xi\left\|A u_{n}-w^{*}\right\|^{2}+\xi\left\|w_{n}-w^{*}\right\|^{2} \\
\leq & \left\|u_{n}-x^{*}\right\|^{2}-\xi\left(1-\xi\left\|A^{*}\right\|^{2}\right)\left\|A u_{n}-w_{n}\right\|^{2}-\xi\left\|A u_{n}-w^{*}\right\|^{2} \\
& +\frac{\xi}{2}\left\|A u_{n}-w^{*}\right\|^{2}+\frac{\xi}{2}\left\|B v_{n}-w^{*}\right\|^{2}
\end{aligned}
$$




$$
\begin{aligned}
= & \left\|u_{n}-x^{*}\right\|^{2}-\xi\left(1-\xi\left\|A^{*}\right\|^{2}\right)\left\|A u_{n}-w_{n}\right\|^{2} \\
& -\frac{\xi}{2}\left\|A u_{n}-w^{*}\right\|^{2}+\frac{\xi}{2}\left\|B v_{n}-w^{*}\right\|^{2}
\end{aligned}
$$

and

$$
\begin{aligned}
\left\|k_{n}-y^{*}\right\|^{2}= & \left\|P_{Q}\left(v_{n}-\xi B^{*}\left(B v_{n}-w_{n}\right)\right)-y^{*}\right\|^{2} \leq\left\|v_{n}-y^{*}-\xi B^{*}\left(B v_{n}-w_{n}\right)\right\|^{2} \\
= & \left\|v_{n}-y^{*}\right\|^{2}+\left\|\xi B^{*}\left(B v_{n}-w_{n}\right)\right\|^{2}-2 \xi\left\langle v_{n}-y^{*}, B^{*}\left(B v_{n}-w_{n}\right)\right\rangle \\
= & \left\|v_{n}-y^{*}\right\|^{2}+\left\|\xi B^{*}\left(B v_{n}-w_{n}\right)\right\|^{2}-2 \xi\left\langle B v_{n}-B y^{*}, B v_{n}-w_{n}\right\rangle \\
= & \left\|v_{n}-y^{*}\right\|^{2}+\left\|\xi B^{*}\left(B v_{n}-w_{n}\right)\right\|^{2}-2 \xi\left\langle B v_{n}-w^{*}, B v_{n}-w_{n}\right\rangle \\
= & \left\|v_{n}-y^{*}\right\|^{2}+\left\|\xi B^{*}\left(B v_{n}-w_{n}\right)\right\|^{2}-\xi\left\|B v_{n}-w^{*}\right\|^{2} \\
& -\xi\left\|B v_{n}-w_{n}\right\|^{2}+\xi\left\|w_{n}-w^{*}\right\|^{2} \\
\leq & \left\|v_{n}-y^{*}\right\|^{2}-\xi\left(1-\xi\left\|B^{*}\right\|^{2}\right)\left\|B v_{n}-w_{n}\right\|^{2}-\xi\left\|B v_{n}-w^{*}\right\|^{2}+\xi\left\|w_{n}-w^{*}\right\|^{2} \\
\leq & \left\|v_{n}-y^{*}\right\|^{2}-\xi\left(1-\xi\left\|B^{*}\right\|^{2}\right)\left\|B v_{n}-w_{n}\right\|^{2}-\xi\left\|B v_{n}-w^{*}\right\|^{2} \\
& +\frac{\xi}{2}\left\|A u_{n}-w^{*}\right\|^{2}+\frac{\xi}{2}\left\|B v_{n}-w^{*}\right\|^{2} \\
= & \left\|v_{n}-y^{*}\right\|^{2}-\xi\left(1-\xi\left\|B^{*}\right\|^{2}\right)\left\|B v_{n}-w_{n}\right\|^{2} \\
& -\frac{\xi}{2}\left\|B v_{n}-w^{*}\right\|^{2}+\frac{\xi}{2}\left\|A u_{n}-w^{*}\right\|^{2} .
\end{aligned}
$$

By taking into account inequalities (3.1), (3.2) and (3.3), we obtain

$$
\begin{aligned}
\left\|l_{n}-x^{*}\right\|^{2}+\left\|k_{n}-y^{*}\right\|^{2} \leq & \left\|u_{n}-x^{*}\right\|^{2}+\left\|v_{n}-y^{*}\right\|^{2}-\xi\left(1-\xi\left\|A^{*}\right\|^{2}\right)\left\|A u_{n}-w_{n}\right\|^{2} \\
& -\xi\left(1-\xi\left\|B^{*}\right\|^{2}\right)\left\|B v_{n}-w_{n}\right\|^{2} \\
\leq & \left\|x_{n}-x^{*}\right\|^{2}+\left\|y_{n}-y^{*}\right\|^{2}-\xi\left(1-\xi\left\|A^{*}\right\|^{2}\right)\left\|A u_{n}-w_{n}\right\|^{2} \\
& -\xi\left(1-\xi\left\|B^{*}\right\|^{2}\right)\left\|B v_{n}-w_{n}\right\|^{2},
\end{aligned}
$$

which implies

$$
\left\|l_{n}-x^{*}\right\|^{2}+\left\|k_{n}-y^{*}\right\|^{2} \leq\left\|u_{n}-x^{*}\right\|^{2}+\left\|v_{n}-y^{*}\right\|^{2} \leq\left\|x_{n}-x^{*}\right\|^{2}+\left\|y_{n}-y^{*}\right\|^{2} .
$$

Inequality (3.5) shows that $\left(x^{*}, y^{*}\right) \in C_{n} \times Q_{n}$. Hence $\Omega \subset C_{n} \times Q_{n}$ and $C_{n} \times Q_{n} \neq \emptyset$ for all $n \in \mathbb{N}$. For each $n \in \mathbb{N}$, since $\Omega \subset C_{n} \times Q_{n}, C_{n+1} \subset C_{n}$, we have

$$
x_{n+1}=P_{C_{n+1}}\left(x_{1}\right) \subset C_{n} .
$$

Similarly, since $Q_{n+1} \subset Q_{n}$, we have

$$
y_{n+1}=P_{Q_{n+1}}\left(y_{1}\right) \subset Q_{n} .
$$

So, for any $\left(x^{*}, y^{*}\right) \in \Omega$, we get

$$
\left\|x_{n+1}-x_{1}\right\| \leq\left\|x^{*}-x_{1}\right\|
$$


and

$$
\left\|y_{n+1}-y_{1}\right\| \leq\left\|y^{*}-y_{1}\right\|
$$

The last inequalities deduce that $\left\{x_{n}\right\}$ and $\left\{y_{n}\right\}$ are bounded and hence show that $\left\{k_{n}\right\}$, $\left\{l_{n}\right\},\left\{u_{n}\right\}$ and $\left\{v_{n}\right\}$ are all bounded. For some $n \in \mathbb{N}$ with $n>1$, from $x_{n}=P_{C_{n}}\left(x_{1}\right) \subset C_{n}$, $y_{n}=P_{Q_{n}}\left(y_{1}\right) \subset Q_{n}$ and (1.1), we have

$$
\begin{aligned}
& \left\|x_{n+1}-x_{n}\right\|^{2}+\left\|x_{1}-x_{n}\right\|^{2}=\left\|x_{n+1}-P_{C_{n}}\left(x_{1}\right)\right\|^{2}+\left\|x_{1}-P_{C_{n}}\left(x_{1}\right)\right\|^{2} \leq\left\|x_{n+1}-x_{1}\right\|^{2}, \\
& \left\|y_{n+1}-y_{n}\right\|^{2}+\left\|y_{1}-y_{n}\right\|^{2}=\left\|y_{n+1}-P_{C_{n}}\left(y_{1}\right)\right\|^{2}+\left\|y_{1}-P_{C_{n}}\left(y_{1}\right)\right\|^{2} \leq\left\|y_{n+1}-y_{1}\right\|^{2},
\end{aligned}
$$

which yields that

$$
\left\|x_{1}-x_{n}\right\| \leq\left\|x_{n+1}-x_{1}\right\|, \quad\left\|y_{1}-y_{n}\right\| \leq\left\|y_{n+1}-y_{1}\right\|
$$

Together with the boundedness of $\left\{x_{n}\right\}$ and $\left\{y_{n}\right\}$, we know $\lim _{n \rightarrow \infty}\left\|x_{n}-x_{1}\right\|$ and $\lim _{n \rightarrow \infty}\left\|y_{n}-y_{1}\right\|$ exist. For some $k, n \in \mathbb{N}$ with $k>n>1$, due to $x_{k}=P_{C_{k}}\left(x_{1}\right) \subset C_{n}$, $y_{k}=P_{Q_{k}}\left(y_{1}\right) \subset Q_{n}$ and (1.1), we have

$$
\begin{aligned}
& \left\|x_{k}-x_{n}\right\|^{2}+\left\|x_{1}-x_{n}\right\|^{2}=\left\|x_{k}-P_{C_{n}}\left(x_{1}\right)\right\|^{2}+\left\|x_{1}-P_{C_{n}}\left(x_{1}\right)\right\|^{2} \leq\left\|x_{k}-x_{1}\right\|^{2}, \\
& \left\|y_{k}-y_{n}\right\|^{2}+\left\|y_{1}-y_{n}\right\|^{2}=\left\|y_{k}-P_{Q_{n}}\left(y_{1}\right)\right\|^{2}+\left\|y_{1}-P_{Q_{n}}\left(y_{1}\right)\right\|^{2} \leq\left\|y_{k}-y_{1}\right\|^{2} .
\end{aligned}
$$

By (3.6), we have $\lim _{n \rightarrow \infty}\left\|x_{n}-x_{k}\right\|=0$ and $\lim _{n \rightarrow \infty}\left\|y_{n}-y_{k}\right\|=0$. Hence $\left\{x_{n}\right\}$ and $\left\{y_{n}\right\}$ are all Cauchy sequences. Let $x_{n} \rightarrow p$ and $y_{n} \rightarrow q$ for some $(p, q) \in C \times Q$. We want to prove that $(p, q) \in \Omega$. For any $n \in \mathbb{N}$, since

$$
\left(x_{n+1}, y_{n+1}\right) \in C_{n+1} \times Q_{n+1} \subset C_{n} \times Q_{n},
$$

from (2.1), we have

$$
\begin{aligned}
\left\|l_{n}-x_{n+1}\right\|^{2}+\left\|k_{n}-y_{n+1}\right\|^{2} & \leq\left\|u_{n}-x_{n+1}\right\|^{2}+\left\|v_{n}-y_{n+1}\right\|^{2} \\
& \leq\left\|x_{n}-x_{n+1}\right\|^{2}+\left\|y_{n}-y_{n+1}\right\|^{2} .
\end{aligned}
$$

By taking the limit from both sides of (3.7), we obtain

$$
\begin{aligned}
& \lim _{n \rightarrow \infty}\left\|l_{n}-x_{n+1}\right\|=\lim _{n \rightarrow \infty}\left\|k_{n}-y_{n+1}\right\|=0, \\
& \lim _{n \rightarrow \infty}\left\|u_{n}-x_{n+1}\right\|=\lim _{n \rightarrow \infty}\left\|v_{n}-y_{n+1}\right\|=0 .
\end{aligned}
$$

Moreover, by (3.8), we get

$$
\begin{aligned}
& \lim _{n \rightarrow \infty}\left\|l_{n}-u_{n}\right\|=\lim _{n \rightarrow \infty}\left\|u_{n}-x_{n}\right\|=\lim _{n \rightarrow \infty}\left\|l_{n}-x_{n}\right\|=0, \\
& \lim _{n \rightarrow \infty}\left\|k_{n}-v_{n}\right\|=\lim _{n \rightarrow \infty}\left\|v_{n}-y_{n}\right\|=\lim _{n \rightarrow \infty}\left\|k_{n}-y_{n}\right\|=0 .
\end{aligned}
$$

Since $\lim _{n \rightarrow \infty}\left\|u_{n}-x_{n}\right\|=\lim _{n \rightarrow \infty}\left\|v_{n}-y_{n}\right\|=0$, we have $u_{n} \rightarrow p$ and $v_{n} \rightarrow q$ as $n \rightarrow \infty$. Moreover, we obtain $A u_{n} \rightarrow A p$ and $B v_{n} \rightarrow B q$ as $n \rightarrow \infty$. 
Now, we claim $p \in \operatorname{EP}(f)$ and $q \in \operatorname{EP}(g)$. In fact, for $r>0$, by Lemma 2.4, we have

$$
\begin{aligned}
\left\|T_{r}^{f} p-p\right\| & =\left\|T_{r}^{f} p-T_{r_{n}}^{f} x_{n}+T_{r_{n}}^{f} x_{n}-x_{n}+x_{n}-p\right\| \\
& \leq\left\|x_{n}-p\right\|+\frac{\left|r_{n}-r\right|}{r_{n}}\left\|T_{r_{n}}^{f} x_{n}-x_{n}\right\|+\left\|T_{r_{n}}^{f} x_{n}-x_{n}\right\|+\left\|x_{n}-p\right\| \\
& =\left\|x_{n}-p\right\|+\frac{\left|r_{n}-r\right|}{r_{n}}\left\|u_{n}-x_{n}\right\|+\left\|u_{n}-x_{n}\right\|+\left\|x_{n}-p\right\| \rightarrow 0
\end{aligned}
$$

and

$$
\begin{aligned}
\left\|T_{r}^{g} q-q\right\| & \leq\left\|T_{r}^{g} q-T_{r_{n}}^{g} y_{n}+T_{r_{n}}^{g} y_{n}-y_{n}+y_{n}-q\right\| \\
& \leq\left\|y_{n}-q\right\|+\frac{\left|r_{n}-r\right|}{r_{n}}\left\|T_{r_{n}}^{g} y_{n}-y_{n}\right\|+\left\|T_{r_{n}}^{g} y_{n}-y_{n}\right\|+\left\|y_{n}-q\right\| \\
& =\left\|y_{n}-q\right\|+\frac{\left|r_{n}-r\right|}{r_{n}}\left\|v_{n}-y_{n}\right\|+\left\|v_{n}-y_{n}\right\|+\left\|y_{n}-q\right\| \rightarrow 0 .
\end{aligned}
$$

So, $p \in \operatorname{EP}(f)$ and $q \in \operatorname{EP}(g)$.

Finally, we prove $A p=B q \in \mathrm{EP}(h)$. Setting

$$
\theta=\min \left\{\xi\left(1-\xi\left\|A^{*}\right\|^{2}\right), \xi\left(1-\xi\left\|B^{*}\right\|^{2}\right)\right\}
$$

Then, for any $n \in \mathbb{N}$, by (3.4) and (3.9), we have

$$
\begin{aligned}
\theta \| & A u_{n}-w_{n}\left\|^{2}+\theta\right\| B v_{n}-w_{n} \|^{2} \\
\leq & \left\|x_{n}-x^{*}\right\|^{2}+\left\|y_{n}-y^{*}\right\|^{2}-\left\|l_{n}-x^{*}\right\|^{2}-\left\|k_{n}-y^{*}\right\|^{2} \\
= & \left\{\left\|x_{n}-x^{*}\right\|-\left\|l_{n}-x^{*}\right\|\right\}\left\{\left\|x_{n}-x^{*}\right\|+\left\|l_{n}-x^{*}\right\|\right\} \\
& +\left\{\left\|y_{n}-y^{*}\right\|-\left\|k_{n}-y^{*}\right\|\right\}\left\{\left\|y_{n}-y^{*}\right\|+\left\|k_{n}-y^{*}\right\|\right\} \\
\leq & \left\|l_{n}-x_{n}\right\|\left\{\left\|x_{n}-x^{*}\right\|+\left\|l_{n}-x^{*}\right\|\right\}+\left\|k_{n}-y_{n}\right\|\left\{\left\|y_{n}-y^{*}\right\|+\left\|k_{n}-y^{*}\right\|\right\} \\
\rightarrow & 0 .
\end{aligned}
$$

Hence (3.10) implies

$$
\lim _{n \rightarrow \infty}\left\|A u_{n}-w_{n}\right\|=\lim _{n \rightarrow \infty}\left\|B v_{n}-w_{n}\right\|=0, \quad \text { and } \quad \lim _{n \rightarrow \infty}\left\|A u_{n}-B v_{n}\right\|=0
$$

Since $A u_{n} \rightarrow A p, B v_{n} \rightarrow B q$ and (3.11), we obtain $A p=B q$ and $w_{n} \rightarrow w^{*}$, where $w^{*}:=A p=$ $B q$. On the other hand, for $r>0$, by Lemma 2.4 again, we have

$$
\begin{aligned}
& \left\|T_{r}^{h} w^{*}-w^{*}\right\| \\
& =\left\|T_{r}^{h} w^{*}-T_{r_{n}}^{h} \frac{A u_{n}+B v_{n}}{2}+T_{r_{n}}^{h} \frac{A u_{n}+B v_{n}}{2}-\frac{A u_{n}+B v_{n}}{2}+\frac{A u_{n}+B v_{n}}{2}-w^{*}\right\| \\
& \leq\left\|\frac{A u_{n}+B v_{n}}{2}-w^{*}\right\|+\frac{\left|r_{n}-r\right|}{r_{n}}\left\|T_{r_{n}}^{h} \frac{A u_{n}+B v_{n}}{2}-\frac{A u_{n}+B v_{n}}{2}\right\| \\
& \quad+\left\|T_{r_{n}}^{h} \frac{A u_{n}+B v_{n}}{2}-\frac{A u_{n}+B v_{n}}{2}\right\|+\left\|\frac{A u_{n}+B v_{n}}{2}-w^{*}\right\|
\end{aligned}
$$




$$
=2\left\|\frac{A u_{n}+B v_{n}}{2}-w^{*}\right\|+\frac{\left|r_{n}-r\right|}{r_{n}}\left\|w_{n}-\frac{A u_{n}+B v_{n}}{2}\right\|+\left\|w_{n}-\frac{A u_{n}+B v_{n}}{2}\right\| \rightarrow 0 .
$$

Hence $w^{*} \in \operatorname{EP}(h)$, namely $A p=B q \in \operatorname{EP}(h)$. Therefore, conclusions (a), (b) and (c) are all proved. The proof is completed.

\section{An example of Theorem 2.1}

In this section, we give an example illustrating Theorem 2.1.

Example 4.1 Let $H_{1}=R^{2}, H_{2}=R^{3}$ and $H_{3}=R^{4}$ be three real Hilbert spaces with the standard norm and inner product. For each $\alpha=\left(\alpha_{1}, \alpha_{2}\right) \in R^{2}$ and $v=\left(z_{1}, z_{2}, z_{3}, z_{4}\right) \in R^{4}$, define

$$
A \alpha=\left(\alpha_{1}, \alpha_{2}, \alpha_{1}+\alpha_{2}, \alpha_{1}-\alpha_{2}\right)
$$

and

$$
A^{*} v=\left(z_{1}+z_{3}+z_{4}, z_{2}+z_{3}-z_{4}\right)
$$

Then $A$ is a bounded linear operator from $R^{2}$ into $R^{4}$ with $\|A\|=\sqrt{3}$, and $A^{*}$ is an adjoint operator of $A$ with $\left\|A^{*}\right\|=\sqrt{3}$. For each $\beta=\left(\beta_{1}, \beta_{2}, \beta_{3}\right) \in R^{3}$ and $v=\left(z_{1}, z_{2}, z_{3}, z_{4}\right) \in R^{4}$, let

$$
B \beta=\left(\beta_{1}, \beta_{2}, \beta_{3}, \beta_{1}-\beta_{2}\right)
$$

and

$$
B^{*} v=\left(z_{1}+z_{4}, z_{2}-z_{4}, z_{3}\right)
$$

Then $B$ is a bounded linear operator from $R^{3}$ into $R^{4}$ with $\|B\|=\sqrt{3}$, and $B^{*}$ is an adjoint operator of $B$ with $\left\|B^{*}\right\|=\sqrt{3}$. Put

$$
\begin{aligned}
& C:=\left\{\alpha=\left(\alpha_{1}, \alpha_{2}\right) \in R^{2}:-1 \leq \alpha_{1} \leq 2,3 \leq \alpha_{2} \leq 4\right\} \\
& Q:=\left\{\beta=\left(\beta_{1}, \beta_{2}, \beta_{3}\right) \in R^{3}:-1 \leq \beta_{1} \leq 1,3 \leq \beta_{2} \leq 4,3 \leq \beta_{3} \leq 5\right\}
\end{aligned}
$$

and

$$
K:=\left\{z=\left(z_{1}, z_{2}, z_{3}, z_{4}\right) \in R^{4}: 0 \leq z_{1} \leq 1,3 \leq z_{2} \leq 6,3 \leq z_{3} \leq 5,-5 \leq z_{4} \leq-3\right\}
$$

For each $\alpha=\left(\alpha_{1}, \alpha_{2}\right) \in C, \beta=\left(\beta_{1}, \beta_{2}, \beta_{3}\right) \in Q$ and $z=\left(z_{1}, z_{2}, z_{3}, z_{4}\right) \in K$, define

$$
\begin{aligned}
& f^{*}(\alpha)=\alpha_{1}^{2}+\alpha_{2}^{2}, \\
& g^{*}(\beta)=\beta_{1}^{2}+\beta_{2}^{2}+\beta_{3}^{2}
\end{aligned}
$$

and

$$
h^{*}(z)=z_{1}^{2}+z_{2}^{2}+z_{3}^{2}+z_{4}^{2} .
$$


For each $\alpha, x \in C$, let

$$
f(\alpha, x)=f^{*}(x)-f^{*}(\alpha)
$$

For each $\beta, y \in Q$, let

$$
g(\beta, y)=g^{*}(y)-g^{*}(\beta)
$$

For each $\eta, z \in K$, let

$$
h(\eta, z)=h^{*}(z)-h^{*}(\eta) .
$$

It is not hard to verify that $f, g$ and $h$ satisfy conditions (A1)-(A4) with $\operatorname{EP}(f)=\{p=(0,3)\}$, $\operatorname{EP}(g)=\{q=(0,3,3)\}, \operatorname{EP}(h)=\{(0,3,3,-3)\}$ and

$$
\Omega=\{(p, q) \in \operatorname{EP}(f) \times \operatorname{EP}(g): A p=B q \in \operatorname{EP}(h)\} \neq \emptyset .
$$

Let $C_{1}=C, Q_{1}=Q, \xi=\frac{1}{6}$ and $r_{n} \equiv 1$ for $n \in \mathbb{N}$. Thus, for each $\bar{x}=(a, b) \in C$ and $\bar{y}=$ $(c, d, e) \in Q$ with $c>0$, we have the following:

- $u=\left(\frac{a}{3}, 3\right)=T_{r_{n}}^{f} \bar{x}$,

- $v=\left(\frac{c}{3}, 3,3\right)=T_{r_{n}}^{g} \bar{y}$,

- $w=\left(\frac{a+c}{6}, 3,3,-3\right)=T_{r_{n}}^{h}\left(\frac{1}{2} A \bar{x}+\frac{1}{2} B \bar{y}\right)$,

- $l=P_{C}\left(u-\frac{1}{6} A^{*}(A u-w)\right)=\left(\frac{7 a+c}{36}, 3\right)$,

- $k=P_{Q}\left(v-\frac{1}{6} B^{*}(B v-w)\right)=\left(\frac{9 c+a}{36}, 3+\frac{c}{18}, 3\right)$.

For $x_{1}=\left(a_{1}, b_{1}\right) \in C$ and $y_{1}=\left(c_{1}, d_{1}, e_{1}\right) \in Q$ with $15 c_{1} \geq a_{1}>0,17 a_{1} \geq c_{1}$ and $d_{1}>3+\frac{c_{1}}{18}$, we obtain the following:

- $u_{1}=\left(\frac{a_{1}}{3}, 3\right)$,

- $v_{1}=\left(\frac{c_{1}}{3}, 3,3\right)$,

- $w_{1}=\left(\frac{a_{1}+c_{1}}{6}, 3,3,-3\right)$,

- $l_{1}=\left(\frac{7 a_{1}+c_{1}}{36}, 3\right)$,

- $k_{1}=\left(\frac{9 c_{1}+a_{1}}{36}, 3+\frac{c_{1}}{18}, 3\right)$,

- $C_{2}=\left\{\alpha=\left(\alpha_{1}, \alpha_{2}\right) \in C_{1}:-1 \leq \alpha_{1} \leq \frac{19 a_{1}+c_{1}}{72}, 3 \leq \alpha_{2} \leq \frac{b_{1}+3}{2}\right\}$,

- $x_{2}=P_{C_{2}}\left(x_{1}\right)=\left(\frac{19 a_{1}+c_{1}}{72}, \frac{b_{1}+3}{2}\right):=\left(a_{2}, b_{2}\right)$,

- $Q_{2}=\left\{\beta=\left(\beta_{1}, \beta_{2}, \beta_{3}\right) \in Q_{1}:-1 \leq \beta_{1} \leq \frac{21 c_{1}+a_{1}}{72}, 3+\frac{c_{1}}{36} \leq \beta_{2} \leq \frac{d_{1}+3}{2}, 3 \leq \beta_{3} \leq \frac{e_{1}+3}{2}\right\}$,

- $y_{2}=P_{Q_{2}}\left(y_{1}\right)=\left(\frac{21 c_{1}+a_{1}}{72}, \frac{d_{1}+3}{2}, \frac{e_{1}+3}{2}\right):=\left(c_{2}, d_{2}, e_{2}\right)$.

From $x_{2}, y_{2}$, we have $u_{2}=\left(\frac{1}{3} a_{2}, 3\right), v_{2}=\left(\frac{1}{3} c_{2}, 3,3\right)$ and $w_{2}=\left(\frac{1}{6}\left(a_{2}+b_{2}\right), 3,3,-3\right)$. Since

$$
\begin{aligned}
& d_{2}>3+\frac{c_{2}}{18}, \\
& 15 c_{2} \geq a_{2}>0,
\end{aligned}
$$

and

$$
17 a_{2} \geq c_{2}
$$


- $C_{3}=\left\{\alpha=\left(\alpha_{1}, \alpha_{2}\right) \in C_{2}:-1 \leq \alpha_{1} \leq \frac{19 a_{2}+c_{2}}{72}, 3 \leq \alpha_{2} \leq \frac{1}{2}\left(b_{2}+3\right)\right\}$,

- $x_{3}=\left(\frac{19 a_{2}+c_{2}}{72}, \frac{1}{2}\left(b_{2}+3\right)\right):=\left(a_{3}, b_{3}\right)$,

- $Q_{3}=\left\{\beta=\left(\beta_{1}, \beta_{2}, \beta_{3}\right) \in Q_{1}:-1 \leq \beta_{1} \leq \frac{21 c_{2}+a_{2}}{72}, 3+\frac{c_{2}}{36} \leq \beta_{2} \leq \frac{d_{2}+3}{2}, 3 \leq \beta_{3} \leq \frac{e_{2}+3}{2}\right\}$,

- $y_{3}=\left(\frac{21 c_{2}+a_{2}}{72}, \frac{d_{2}+3}{2}, \frac{e_{2}+3}{2}\right):=\left(c_{3}, d_{3}, e_{3}\right)$.

Similarly, for $n \in \mathbb{N}$ with $n>1$, we obtain

- $C_{n+1}=\left\{\alpha=\left(\alpha_{1}, \alpha_{2}\right) \in C_{n}:-1 \leq \alpha_{1} \leq \frac{19 a_{n-1}+c_{n-1}}{72}, 3 \leq \alpha_{2} \leq \frac{1}{2}\left(b_{n-1}+3\right)\right\}$,

- $x_{n+1}=\left(\frac{19 a_{n-1}+c_{n-1}}{72}, \frac{1}{2}\left(b_{n-1}+3\right)\right)$,

- $Q_{n+1}=\left\{\beta=\left(\beta_{1}, \beta_{2}, \beta_{3}\right) \in Q_{n}:-1 \leq \beta_{1} \leq \frac{21 c_{n-1}+a_{n-1}}{72}, 3+\frac{c_{n-1}}{36} \leq \beta_{2} \leq \frac{d_{n-1}+3}{2}, 3 \leq \beta_{3} \leq\right.$ $\left.\frac{e_{n-1}+3}{2}\right\}$,

- $y_{n+1}=\left(\frac{21 c_{n-1}+a_{n-1}}{72}, \frac{d_{n-1}+3}{2}, \frac{e_{n-1}+3}{2}\right)$,

- $u_{n+1}=\left(\frac{1}{3} a_{n-1}, 3\right)$,

- $v_{n+1}=\left(\frac{1}{3} c_{n-1}, 3,3\right)$,

- $w_{n+1}=\left(\frac{c_{n-1}+a_{n-1}}{6}, 3,3,-3\right)$.

By mathematical induction, we know that $\left\{a_{n}\right\},\left\{b_{n}\right\},\left\{c_{n}\right\},\left\{d_{n}\right\}$ and $\left\{e_{n}\right\}$ all are decreasing sequences. Moreover, $a_{n} \rightarrow 0, b_{n} \rightarrow 3, c_{n} \rightarrow 0, d_{n} \rightarrow 3$ and $e_{n} \rightarrow 3$ as $n \rightarrow \infty$. So, we have $u_{n} \rightarrow(0,3), v_{n} \rightarrow(0,3,3), w_{n} \rightarrow(0,3,3,-3), x_{n} \rightarrow(0,3)$ and $y_{n} \rightarrow(0,3,3)$ as $n \rightarrow \infty$.

\section{Conclusion}

In this paper, we first introduce and investigate BSEP which can be regarded as a new development in the field of equilibrium problems. We provide some new iterative algorithms for BSEP and establish strong convergence theorems for these iterative algorithms in different spaces. An example illustrating Theorem 2.1 is also given.

\section{Competing interests}

The authors declare that they have no competing interests.

\section{Authors' contributions}

Both authors contributed equally and significantly in writing this paper. Both authors read and approved the final manuscript.

\section{Author details}

'Department of Mathematics, Honghe University, Yunnan, 661100, China. ${ }^{2}$ Department of Mathematics, National Kaohsiung Normal University, Kaohsiung, 824, Taiwan.

\section{Acknowledgements}

The first author was supported by the Natural Science Foundation of Yunnan Province (201401CA00262) and the Candidate Foundation of Youth Academic Experts at Honghe University (2014HB0206); the second author was supported by Grant No. MOST 103-2115-M-017-001 of the Ministry of Science and Technology of the Republic of China.

Received: 1 June 2014 Accepted: 18 August 2014 Published: 02 Sep 2014

\section{References}

1. He, Z: The split equilibrium problems and its convergence algorithms. J. Inequal. Appl. 2012, 162 (2012)

2. Blum, E, Oettli, W: From optimization and variational inequalities to equilibrium problems. Math. Stud. 63, 123-145 (1994)

3. Flam, SD, Antipin, AS: Equilibrium programming using proximal-link algorithms. Math. Program. 78, $29-41$ (1997)

4. Moudafi, A, Théra, M: Proximal and dynamical approaches to equilibrium problems. In: III-Posed Variational Problems and Regularization Techniques. Lecture Notes in Economics and Mathematical Systems, vol. 477, pp. 187-201. Springer, Berlin (1999)

5. Moudafi, A: Second-order differential proximal methods for equilibrium problems. J. Inequal. Pure Appl. Math. 18(4), $1-17$ (2003)

6. Lin, L-J, Du, W-S: Systems of equilibrium problems with applications to new variants of Ekeland's variational principle, fixed point theorems and parametric optimization problems. J. Glob. Optim. 40, 663-677 (2008)

7. Du, W-S: Hybrid inclusion and disclusion systems with applications to equilibria and parametric optimization. J. Glob. Optim. 47, 119-132 (2010)

8. Du, W-S: Applications of an HIDS theorem to the existence of fixed point, abstract equilibria and optimization problems. Abstr. Appl. Anal. 2011, Article ID 247236 (2011). doi:10.1155/2011/247236 
9. Ceng, LC, Yao, JC: Hybrid viscosity approximation schemes for equilibrium problems and fixed point problems of infinitely many nonexpansive mappings. Appl. Math. Comput. 198, 729-741 (2008)

10. Combettes, PL, Hirstoaga, A: Equilibrium programming in Hilbert spaces. J. Nonlinear Convex Anal. 6, 117-136 (2005)

11. He, Z: A new iterative scheme for equilibrium problems and fixed point problems of strict pseudo-contractive mappings and its application. Math. Commun. 17, 411-422 (2012)

12. He, Z, Du, W-S: Strong convergence theorems for equilibrium problems and fixed point problems: a new iterative method, some comments and applications. Fixed Point Theory Appl. 2011, 33 (2011)

13. Jung, JS: Strong convergence of composite iterative methods for equilibrium problems and fixed point problems. Appl. Math. Comput. 213, 498-505 (2009)

14. Tada, A, Takahashi, W: Weak and strong convergence theorems for a nonexpansive mapping and an equilibrium problem. J. Optim. Theory Appl. 133, 359-370 (2007)

15. Takahashi, S, Takahashi, W: Viscosity approximation methods for equilibrium problems and fixed point problems in Hilbert spaces. J. Math. Anal. Appl. 331, 506-515 (2007)

16. Yao, Y, Noor, MA, Liou, YC: On iterative methods for equilibrium problems. Nonlinear Anal. 70, 497-509 (2009)

17. Colao, V, Marino, G: Strong convergence for a minimization problem on points of equilibrium and common fixed points of an infinite family of nonexpansive mappings. Nonlinear Anal. 73, 3513-3524 (2010)

18. Censor, Y, Gibali, A, Reich, S: Algorithms for the split variational inequality problem. Numer. Algorithms 59, $301-323$ (2012)

19. He, Z, Du, W-S: Nonlinear algorithms approach to split common solution problems. Fixed Point Theory Appl. 2012, $130(2012)$

20. He, Z, Du, W-S: On hybrid split problem and its nonlinear algorithms. Fixed Point Theory Appl. 2013, $47(2013)$

21. Moudafi, A: The split common fixed-point problem for demicontractive mappings. Inverse Probl. 26, 055007 (2010)

22. Moudafi, A: A note on the split common fixed-point problem for quasi-nonexpansive operators. Nonlinear Anal. 74, 4083-4087 (2011)

23. Moudafi, A: A relaxed alternating CQ-algorithm for convex feasibility problems. Nonlinear Anal. 79, 117-121 (2013)

24. $\mathrm{Xu}, \mathrm{H}-\mathrm{K}$ : Iterative methods for the split feasibility problem in infinite-dimensional Hilbert spaces. Inverse Probl. 26, 105018 (2010)

25. Chang, SS, Lee, HWJ, Chan, CK: A new method for solving equilibrium problem fixed point problem and variational inequality problem with application to optimization. Nonlinear Anal. 70, 3307-3319 (2009)

10.1186/1687-1812-2014-187

Cite this article as: He and Du: New feasible iterative algorithms and strong convergence theorems for bilevel split equilibrium problems. Fixed Point Theory and Applications 2014, 2014:187

\section{Submit your manuscript to a SpringerOpen ${ }^{\circ}$ journal and benefit from:}

- Convenient online submission

Rigorous peer review

- Immediate publication on acceptance

- Open access: articles freely available online

- High visibility within the field

- Retaining the copyright to your article 